\section{Otorrinolaringología después de la pandemia de COVID-19}

\section{Otorhinolaryngology after COVID-19 pandemic}

\author{
Paul Délano R. ${ }^{1}$
}

${ }^{1}$ Editor. Revista de Otorrinolaringología y Cirugía de Cabeza y Cuello. Departamento de Otorrinolaringología. Hospital Clínico de la Universidad de Chile. Santiago, Chile.

Correspondencia:

Dr. Carlos Lorca Tobar, 999

Servicio de Otorrinolaringología. Hospital

Clínico Universidad de Chile.

Independencia. Santiago, Chile.

Email: pdelano@med.uchile.cl
En junio de 2021 se cumple un año y tres meses desde que la Organización Mundial de la Salud declaró la pandemia mundial por SARS-CoV-2, afectando de manera significativa nuestras relaciones interpersonales, tanto a nivel familiar, de amistades y laborales. La especialidad de otorrinolaringología no ha estado exenta a estos cambios, lo que ha perturbado en forma drástica la práctica clínica y la enseñanza de nuestra disciplina.

Desde el inicio de esta pandemia se han suspendido o retrasado cirugías electivas y atenciones ambulatorias. $\mathrm{Al}$ mismo tiempo, muchos otorrinolaringólogos o residentes han sido reasignados en sus funciones hacia el manejo de pacientes con COVID-19 ${ }^{1}$. Estos cambios han generado largas listas de esperas para atenciones médicas y cirugías en otorrinolaringología. Para afrontar esta situación, se han utilizado diversas estrategias, como por ejemplo, la implementación de programas de telemedicina o la priorización de cirugías oncológicas y de riesgo vital ${ }^{2-4}$. Por otro lado, debido a la necesidad de contar con camas disponibles para pacientes con COVID-19, muchas de nuestras cirugías han debido realizarse de manera ambulatoria, con hospitalizaciones transitorias de unas pocas horas de duración.

La pandemia también ha afectado la docencia en otorrinolaringología, disminuyendo drásticamente las oportunidades quirúrgicas de los médicos en formación ${ }^{1}$. Esto ha acelerado el desarrollo de tecnologías innovadoras para el aprendizaje, como el uso de realidad virtual o de modelos para la enseñanza quirúrgica. También se ha consolidado el formato virtual para las reuniones clínicas, ya que facilita la asistencia de los profesionales, al disminuir los tiempos de traslados entre diferentes lugares de trabajo. Por otro lado, durante la pandemia, se ha producido un aumento exponencial de las oportunidades de acceso a cursos en modalidad online, incluyendo webinars, congresos de especialidad y reuniones con expertos internacionales como, por ejemplo, comités oncológicos y de implantes cocleares. Estas actividades han sido especialmente útiles para el desarrollo de la disciplina en lugares alejados o con pocos recursos, como países africanos y latinoamericanos ${ }^{5}$.

Gracias al rápido desarrollo de vacunas para el SARS-CoV-2, y a la implementación de programas masivos de vacunación a nivel mundial, todos esperamos que el fin de la pandemia ocurra en un futuro próximo. Sin embargo, los programas de vacunación han avanzado más lento de lo esperado, y en forma desigual en los diferentes países. Una vez que la vacunación alcance a una gran proporción de la población mundial -probablemente en los próximos años- se podrá controlar la pandemia, o al menos mantenerla en niveles lo suficientemente bajos, para no poner en riesgo de colapso a los sistemas de salud mundiales.

Es probable que muchos de los cambios que se debieron implementar con la pandemia del SARS-CoV-2 se mantengan en el tiempo. Por ejemplo, el uso de la telemedicina permitirá afrontar las largas listas de espera y mejorar la calidad de la atención de los pacientes ${ }^{2,3}$. Por otro lado, las reuniones científicas podrán desarrollarse en formatos híbridos, manteniendo muchas de las herramientas virtuales como las videoconferencias, pero con actividades presenciales que nos permitan las interacciones directas en el ámbito social y académico. 
La Sociedad Chilena de Otorrinolaringología, Medicina y Cirugía de Cabeza y Cuello (SOCHIORL) tiene planificado realizar el $77^{\circ}$ Congreso Chileno de Otorrinolaringología entre el 9 y 12 de noviembre del 2021 en formato híbrido (www.orl2021-difusion.cl), incluyendo actividades online que se transmitirán a todo el mundo por videoconferencias y talleres presenciales con cupos limitados en las localidades de Pirque, La Serena y Punta Arenas en Chile. Organizar esta actividad científica y social en formato híbrido será, sin duda, un desafío enorme, al cual estamos todos invitados, ya sea en forma presencial o virtual según preferencia o disponibilidad.

Una vez que termine la pandemia del SARS-CoV-2, debemos aprovechar las ventajas de las nuevas herramientas virtuales, con el fin de mejorar la calidad de vida de nuestros pacientes y para optimizar la enseñanza de nuestra especialidad a las futuras generaciones.

\section{Referencias}

1. Álvarez ML, Waissbluth S, González C, et al. How the COVID-19 pandemic affects specialty training: An analysis of a nationwide survey among otolaryngology residents in Chile. How the COVID-19 pandemic affects specialty training: An analysis of a nationwide survey among otolaryngology residents in Chile. Medwave. 2021;21(1):e8098. doi: 10.5867/ medwave.2021.01.8097.

2. Mena C, Toso S, Tornaco R, Pires F, Krauskopf A. Teleconsulta en otorrinolaringología durante la pandemia por COVID-19, nuestra experiencia. Hospital de Clínicas. San Lorenzo, Paraguay 2020. Rev Otorrinolaringol Cir Cabeza Cuello. 2020; 80(4): 411-416. doi: 10.4067/S0718-48162020000400411.

3. García-Huidobro F, Willson M, Sepúlveda V, Palma S, Cabello P. Evaluación de satisfacción en la implementación de un nuevo sistema de telemedicina en el Servicio de Otorrinolaringología del Complejo Asistencial Dr. Sótero del Río (CASR) durante la pandemia de COVID-19. Rev Otorrinolaringol Cir Cabeza Cuello. 2020;80(4), 403-410. doi: 10.4067/ S0718-48162020000400403.

4. Bolívar M, Niebla J, Celis E, Pamanes A, Cázarez M, Osuna B, et al. Creación de un comité de vía aérea quirúrgica para la decisión de traqueostomía electiva en pacientes críticos COVID-19. Rev Otorrinolaringol Cir Cabeza Cuello. 2021;81(1):83-92. doi: 10.4067/ S0718-48162021000100083.

5. Quraishi N, Quraishi S. Otolaryngology education and training in the COVID-19 and post-COVID-19 digital era: a developing world perspective. Curr Opin Otolaryngol Head Neck Surg. 2021;29(3):225-229. doi: 10.1097/MOO.0000000000000709. 